\title{
Exploratory Analysis of Biological Networks through Visualization, Clustering, and Functional Annotation in Cytoscape
}

\author{
Anastasia Baryshnikova ${ }^{1}$ \\ Lewis-Sigler Institute for Integrative Genomics, Princeton University, Princeton, New Jersey 08544
}

Biological networks define how genes, proteins, and other cellular components interact with one another to carry out specific functions, providing a scaffold for understanding cellular organization. Although in-depth network analysis requires advanced mathematical and computational knowledge, a preliminary visual exploration of biological networks is accessible to anyone with basic computer skills. Visualization of biological networks is used primarily to examine network topology, identify functional modules, and predict gene functions based on gene connectivity within the network. Networks are excellent at providing a bird's-eye view of data sets and have the power of illustrating complex ideas in simple and intuitive terms. In addition, they enable exploratory analysis and generation of new hypotheses, which can then be tested using rigorous statistical and experimental tools. This protocol describes a simple procedure for visualizing a biological network using the genetic interaction similarity network for Saccharomyces cerevisiae as an example. The visualization procedure described here relies on the open-source network visualization software Cytoscape and includes detailed instructions on formatting and loading the data, clustering networks, and overlaying functional annotations.

\section{MATERIALS}

Equipment

BiNGO (Maere et al. 2005) or other relevant app for network annotation (see Step 17) Computer system

System requirements are available at www.cytoscape.org/documentation_users.html.

Cytoscape 3.0 (Smoot et al. 2011) (see Step 1)

Network data to be analyzed or sample data (see Steps 2 and 3)

METHOD

Install Cytoscape and Prepare Data

1. Download Cytoscape 3.0 from www.cytoscape.org/download.php. Instructions for installation are available at www.cytoscape.org/documentation_users.html. Proceed to either Step 2 (if using sample data) or Step 3.

\footnotetext{
${ }^{1}$ Correspondence: abarysh@princeton.edu

(C) 2016 Cold Spring Harbor Laboratory Press

Cite this protocol as Cold Spring Harb Protoc; doi:10.1101/pdb.prot077644
} 
2. Download the following sample data from www.baryshnikova-lab.org/publications/4/.

- Data file 1. A tab-delimited text file containing the genetic interaction similarity data from Costanzo et al. (2010): Costanzo_Science_2010_correlation_network.txt.

This file contains a list of gene pairs (Columns 1 and 2) whose genetic interaction profile similarity, as measured by Pearson correlation coefficient, is $>0.2$ (Column 3). Here, a genetic interaction is defined as an unexpected phenotype arising from combining two mutations in the same organism. In Costanzo et al. (2010), genetic interactions were quantitatively measured for $30 \%$ of all possible double-mutant combinations in the yeast Saccharomyces cerevisiae. Genes sharing similar genetic interactions are known to share similar functions. We can therefore build a network of functional relationships between all genes in the data set by computing a quantitative measure of profile similarity, such as Pearson correlation, and selecting the gene pairs with the highest observable correlation.

- Data file 2. A tab-delimited text file containing the functional annotation standard used for visualization in Figure 1 in Costanzo et al. (2010): Costanzo_Science_2010_functional_annotation.txt.

This file contains the list of genes in the Costanzo et al. network and four different node attributes: CommonName (Column 2; the three-letter code for the gene), ORF (Column 3; the systematic name for the gene), FunctionalAnnotation (Column 4; discrete values indicating the association of the gene with one of 13 different functional groups; see Step 18), and Essentiality (Column 5; binary 0-1 values indicating whether the gene is known to be essential or not). Column 1 contains a unique StrainID that matches the node label used in Data file 1.

3. (Optional) If not using sample data, prepare network data for import.

Network data can be imported into Cytoscape using a variety of file formats (wiki.cytoscape.org/ Cytoscape_3/UserManual/Network_Formats/). The simplest format is a delimited text file that defines a network by listing its interactions (Fig. 1A; Data file 1). Each interaction (or "edge") must report the labels of the two connected nodes (i.e., gene or protein names) and, optionally, may also include any number of interaction properties (or "edge attributes"), such as type, strength, and confidence of the interaction. Columns containing node labels and edge attributes must be separated by a special character (e.g., a tab or a comma) that is never encountered within a node label (Fig. 1A; Data file 1).

The delimited text file is the simplest way to store and exchange network data because it can be generated and edited in many applications on many platforms and is easily interpretable by both humans and computers. The limitation of delimited text files is that they do not carry any information relative to the positioning of nodes in the network and do not specify any node attribute, such as alternative labels, shape, color, or annotation. This information, if available, must be stored in an additional file and loaded separately (see Step 18i-Loading node attributes). More complex file formats, such as XGMML, store all network information in a single file and allow a user to import organized and annotated networks in a single step. XGMML files, however, must be generated using a specialized tool, involve relatively complex syntax (http://cgi5.cs.rpi .edu/research/groups/pb/punin/public_html/XGMML/), and are not easily manipulated. As a result, $X G M M L$ files are more appropriate for advanced users to exchange visualizations across different applications or across different networks within the same application.

\section{Load the Network}

4. In Cytoscape, go to File $\rightarrow$ Import $\rightarrow$ Network $\rightarrow$ File. Choose the file containing the network data and click Open.

In the sample data provided, the file is Costanzo_Science_2010_correlation_network.txt.

5. View the Preview section of the window, which displays a sample of the input file and shows how information in the file will be parsed.

6. If parsing appears incorrect (e.g., two node labels are merged in the same column or, conversely, a single node label is split into multiple columns), click on Show Text File Import Options in the Advanced section of the window to specify a different column delimiter character. In addition, indicate whether the input file contains any header rows (Start Import Row) and/or whether the first row should be used to label the columns (Transfer first line as column names).

7. In the Interaction Definition section of the window, specify which columns contain the labels of the interacting nodes (Source Interaction and Target Interaction).

The corresponding columns in the Preview section will be highlighted in purple and orange, respectively. 
A

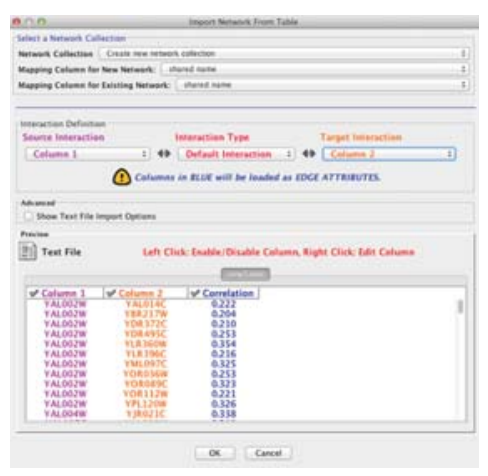

C

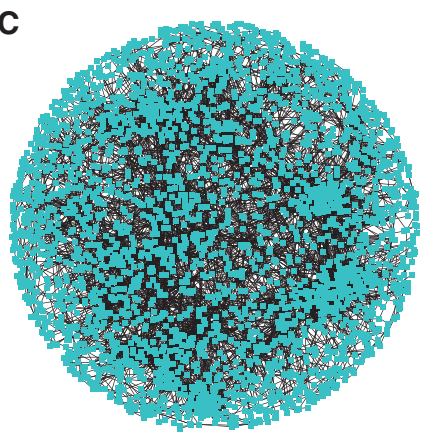

B

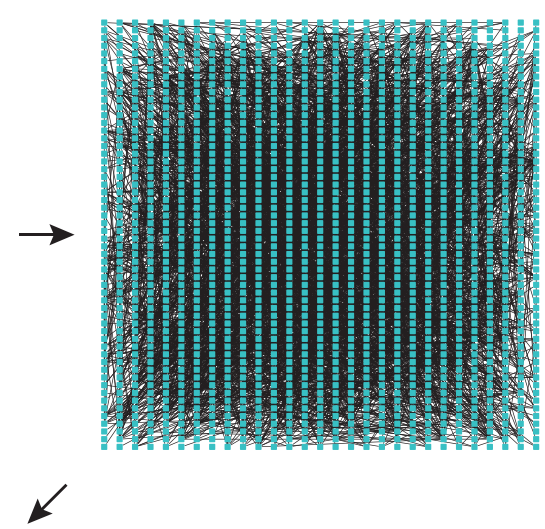

D

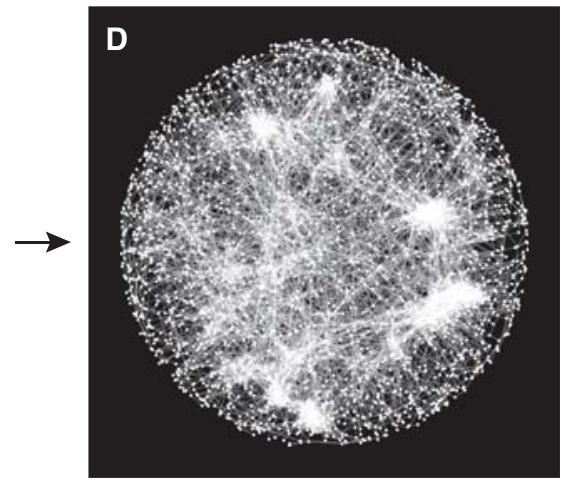

E
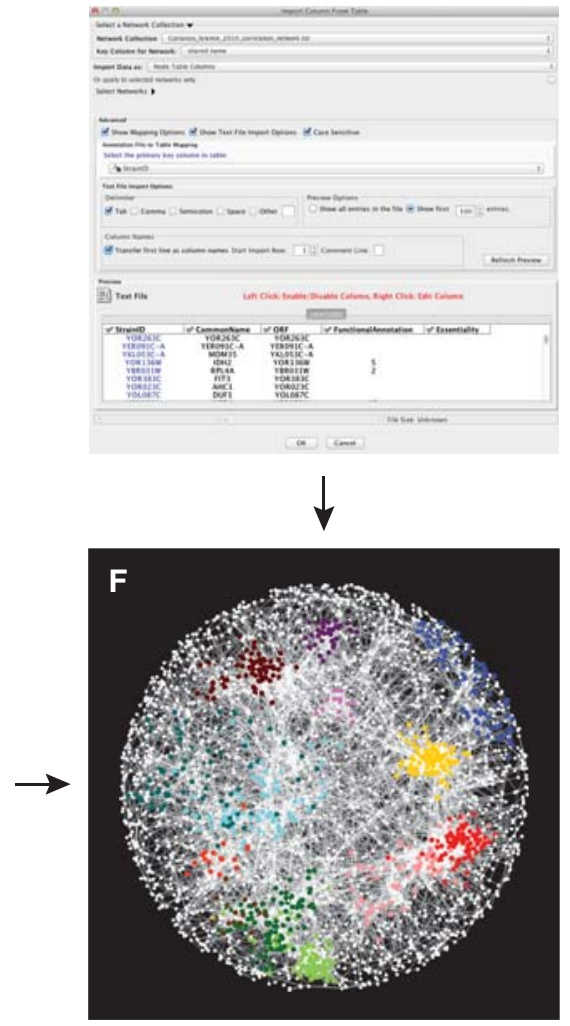

FIGURE 1. Key steps for visualizing a large-scale biological network using Cytoscape 3.0. (A) Loading the network data from a tab-delimited text file. $(B)$ Creating a preliminary view of the network. $(C)$ Organizing the network using the edge-weighted spring-embedded layout. $(D)$ Adjusting the visual appearance of the network by changing color, shape, size, and transparency of nodes and edges. $(E)$ Loading customized functional annotations as node attributes. $(F)$ Adjusting node appearance based on node attributes.

For undirected edges (e.g., protein-protein interactions), it does not matter which node is labeled as Source and which one as Target, unless, for example, it is important to preserve the order in which the interaction was detected (Source and Target may correspond to bait and prey proteins, respectively). For directed edges (e.g., kinase-substrate relationships), Source and Target should indicate the upstream (kinase) and downstream (substrate) nodes, respectively.

In the example provided (undirected edges), Columns 1 and 2 should be labeled as Source and Target (or vice versa) (Fig. 1A).

8. Click on the columns containing edge attributes to select them. Each selected column will be highlighted in blue. To name an attribute and/or to specify whether it contains texts or numerical values, right-click (or Command-click in Mac OS X) on the column and input the relevant information.

In the example provided, Column 3 contains a measure of similarity (Pearson correlation coefficients) between the genetic interaction profiles of Source and Target genes. This attribute should be named Correlation and assigned to the Floating Point data type (Figure 1A). Alternatively, if, for example, this column contained a discrete score (from 1 to 5) representing our level of confidence in the correlation, we would have named the edge attribute Confidence and assigned it to the Integer data type.

9. Click OK to load the network.

10. If the network does not appear automatically, right-click on the name of the network in the Control Panel on the left and select Create View.

This step is usually necessary for large networks because they are not automatically visualized to preserve memory. 
A. Baryshnikova

In the sample data provided, the Control Panel should list a network named Costanzo_Science_2010_correlation_network.txt with 2838 nodes and 10,189 edges (Fig. 1B).

11. Once the network has been loaded and visualized, save it as a Cytoscape Session file (*.cys), which can then be reopened at a later time on the same or on a different computer and will maintain all the properties (layout, annotations, and visual style) of the original network.

\title{
Organize the Network
}

For a discussion of possible ways to organize the network see the Discussion section.

12. As an example, we here apply the edge-weighted spring-embedded layout, which mimics the behavior of a system of connected springs and reorganizes the network such that densely connected nodes are positioned close to each other, whereas disconnected nodes are spread farther apart. To apply the layout, select Layout $\rightarrow$ Edge-weighted Spring Embedded. As an option, an edge attribute can be used to specify the spring force coefficient. In our example, choose the Correlation node attribute to apply stronger forces to highly correlated gene pairs or select (none) to assign equal forces to all gene pairs.

\begin{abstract}
Because of a randomization step in the algorithm, the edge-weighted spring-embedded layout generates a new network configuration at every run. Although these configurations are equivalent in terms of force balance between the edges connecting each node, it is not possible to reproduce the same exact network visualization in two independent Cytoscape sessions.
\end{abstract}

\section{Adjust the Visual Style of the Network}

The procedure described below reproduces the visual style of the correlation network published as Fig. 1 in Costanzo et al. (2010). It is provided as an example of the visual properties that can be adjusted in Cytoscape to generate informative network visualizations (Fig. 1D). Additional options can be explored by the user within Cytoscape.

13. Set the background color to black.

i. Go to Control Panel $\rightarrow$ VizMapper. Click on the image in the Defaults (Click to edit) section.

ii. Click on Network at the bottom right of the window. Double-click on Network Background Paint and choose the black color.

14. Make the edges white and semitransparent.

i. In the same window as above, click on Edge at the bottom right of the window.

ii. In the bottom center, click on Show All.

iii. Scroll through the properties to find Edge Stroke Color (Unselected), double-click, and choose the white color.

iv. Scroll through the properties to find Edge Transparency, double-click, and input 50 (values 0 and 255 correspond to $0 \%$ and $100 \%$ opacity, respectively).

15. Make the nodes white, small, and round.

i. In the same window as above, click on Node at the bottom right of the window.

ii. In the bottom center, click on Show All.

iii. Scroll through the properties to find Node Size, double-click, and input 20.

iv. Scroll through the properties to find Node Fill Color, double-click, and choose the white color.

v. Scroll through the properties to find Node Shape, double-click, and choose Ellipse.

16. Click on Apply in the bottom left corner of the window (Fig. 1D).

Depending on the network size, some of the visual changes might not be immediately visible in the Cytoscape main window. To see the changes, it may be necessary to zoom in or out of the current network view ("+" and "-" magnifying glasses in the top toolbar) or export the current network view into a PDF file (see Step 19). 


\section{Annotate the Network}

For a discussion of how and why to use network annotation, see the Discussion section.

17. Perform functional enrichment.

While functional enrichment tests are not directly implemented in the default version of Cytoscape, numerous third-party apps have been developed that provide this capability (http://apps.cytoscape.org/apps/ with_tag/enrichmentanalysis). As an example, I describe the app BiNGO (Maere et al. 2005) with most of its default settings. BiNGO is particularly suitable for functional annotation of biological networks because it enables the user to test selected network regions for enrichment against common functional annotation standards, such as Gene Ontology biological process terms, and returns a standard significance p-value.

i. To download and install BiNGO, choose the menu Apps $\rightarrow$ App Manager. In the Search window, type BiNGO, select the app from the list, and click on Install.

ii. To run a functional enrichment analysis, select a subset of nodes (e.g., a cluster of interest) from the network and choose the menu Apps $\rightarrow$ BiNGO.

iii. Type in a name for the selected cluster.

By default, BiNGO extracts node labels directly from the network (Get Cluster from Network, selected by default) and compares them against the specified Gene Ontology file (lower in this window, Select ontology file: GO_Biological_Process, GO_Cellular_Component, GOSlim_Yeast, etc.). Some of the node labels, however, may not match the gene identifiers listed in GO due to the existence of gene name aliases (e.g., SRS2 or HPR5 for YJLO92W) and/or custom annotations appended to node labels (e.g., in our example, YAL041W_tsq148 denotes one of the several CDC24 temperature-sensitive alleles present in the data set). In theory, it is possible to provide an additional node attribute containing a standard gene identifier for all nodes in the network (see Step 18i-Loading node attributes). However, BiNGO cannot perform functional enrichment on node attributes. Instead, standard gene identifiers corresponding to the selected nodes can be manually copied from the network window (or any other source) and pasted directly into the BiNGO settings window (following the selection of the Paste Genes from Text option).

iv. Leave all other options as their defaults (for more details, see Maere et al. (2005)).

v. Click Start BiNGO.

vi. View results.

The results window lists the GO terms whose members are significantly overrepresented/enriched among the genes in the selected cluster. In addition, BiNGO generates a network visualizing the distribution of significantly overrepresented GO terms in the overall hierarchy of GO.

18. Perform visual annotation using functional standards.

In Cytoscape, node attributes (i.e., numerical values or text labels associated with each node) can be used to associate nodes with unique visual properties, such as size, shape, and color. Using this feature, genes acting in known biological pathways can be easily identified and assessed qualitatively with respect to network topology. For example, we can determine whether members of a biological pathway cluster together in a network module or whether essential genes tend to be highly connected and act as network hubs.

i. Check the file containing node attribute data: Similarly to network data, the simplest format for importing node attributes is a delimited text file where each row corresponds to a node and each column corresponds to a different node attribute. Columns must be separated by a special character (e.g., a tab or a comma) (Fig. 1E; Data file 2). One of the columns must contain a node identifier: the primary identifier used in the network data file (see Step 3) or a secondary identifier previously loaded as a node attribute (e.g., see Step 18v).

In our example (Fig. 1E), the node attribute file contains a customized functional annotation: genes are assigned a functional group (numbered from 1 to 13) if they (a) belong to one of the large functionally enriched clusters in the network and (b) are annotated to the biological process most represented in that cluster. These numbers were generated specifically for the genetic interaction similarity network using a multistep procedure involving network clustering, functional enrichment, and manual annotation (Costanzo et al. 2010).

ii. Go to File $\rightarrow$ Import $\rightarrow$ Table $\rightarrow$ File. Choose the file containing the node attribute data and click Open.

iii. View the Preview section of the window, which displays a sample of the input file showing how it will be parsed for information. 
A. Baryshnikova

iv. If parsing appears incorrect, click on Show Text File Import Options in the Advanced section of the window to specify a different column delimiter character. In addition, you can indicate whether or not the first row of the file should be used to label the columns.

v. To select a different node label in the network, click on the Key Column for Network dropdown menu (second from the top of the window) and select the desired node label.

By default, Cytoscape will match the first column of the node attribute file to the primary node label in the network. Both of these options can be changed.

It may be necessary to load additional node labels before being able to load the functional annotation. For example, the primary node identifier in the network may be the common name of a gene, whereas the functional annotation standard lists genes by their systematic ORF (open reading frame) names. As a result, it is first necessary to create a node attribute containing the systematic ORF name corresponding to each common name label. This can be done using the same exact procedure described in this section (Steps $i-v$ ). Once the node attribute containing the systematic ORF name has been created, it can be used as Key Column for Network to load the functional annotation standard.

vi. To select a different column from the node attribute file, click on Show Mapping Options and select the correct column from the Select the primary key column in table drop-down menu. The corresponding column will be highlighted in blue in the Preview section.

vii. By default, all columns of the node attribute file will be loaded as attributes and will be named Column 1, Column 2, Column 3, etc. To avoid loading unnecessary information, click on a column in the Preview section to deselect it. To rename an attribute and/or specify whether it contains text or numerical values, right-click (or Command-click in Mac OS X) on the column and input the relevant information.

viii. To load the node attribute file, click OK.

ix. To assign visual properties to node attributes (Fig. 1F): Go to Control Panel $\rightarrow$ VizMapper.

x. In the Visual Mapping Browser section, click on Show All. Scroll down all the Node and Edge properties and double-click on Node Fill Color.

xi. Click on Please select a value and choose the node attribute that should be represented as a color.

In our example, choose the FunctionalAnnotation node attribute.

xii. Click on the cell next to Mapping Type and select Discrete Mapping.

Discrete Mapping will associate a distinct color to each of the unique values in the FunctionalAnnotation node attribute. As a result, if the network contains 10 nodes annotated to functional group 1 and this functional group is associated with the color red, the network will show 10 red nodes.

xiii. Right-click (or Command-click in Mac OS X) on Node Fill Color, select Mapping Value Generators $\rightarrow$ Rainbow (or any other option). This will assign random colors to the functional annotation groups.

xiv. To adjust a color associated with a specific functional group, click on the category in Vizmapper, then click on .... and select the appropriate color.

xv. Similarly, node attributes can be associated with different node shape, size, and transparency.

19. Finalize network visualization: It is often convenient to manually adjust the visualization of a network using a vector graphic editor such as Adobe Illustrator. To do this, export the network from Cytoscape into an editable PDF file by choosing File $\rightarrow$ Export $\rightarrow$ Network View as Graphics $\rightarrow$ PDF File (*.pdf). Click on Save a File to specify the name and the location of the PDF file.

DISCUSSION

Network Organization

Cytoscape provides a wide range of automatic network layout options (http://wiki.cytoscape.org/ Cytoscape_3/UserManual\#Cytoscape_3.2BAC8-UserManual.2BAC8-Navigation_Layout.Automatic_ 
Network Annotation

To evaluate a network and determine how well it recapitulates known biology, it is often useful to visually associate nodes and/or node clusters with specific biological processes. For example, a functional enrichment test may be required to determine whether members of a particular network cluster are overrepresented/enriched for genes annotated to a particular Gene Ontology term. Gene Ontology (GO) is a standardized vocabulary that describes every gene's biological role, molecular function, and cellular localization (Ashburner et al. 2000), and provides a unique resource for evaluating the output of genomic experiments and large scale networks (see Step 17-Functional enrichment). As an alternative to functional enrichment and GO, the user may consider directly labeling the network using a custom set of functional annotations such as, for example, a set of mutants with a phenotype of interest or a hit list from a systematic experiment, and visually assessing the distribution of functional groups throughout the network (see Step 18-Visual annotation using functional standards). This latter approach, while less rigorous, often provides a rapid and useful overview of functional information within the network that may motivate the implementation of more accurate statistical tests.

The level of detail with which a network should be annotated depends on the purpose of the visualization and on the availability of properly defined annotation standards. Although GO is certainly the most widely adopted standard, its hierarchical structure often hinders its direct use in network visualization without preliminary manipulation, such as compression and/or filtering (e.g., Myers et al. 2006). One potential alternative is GO Slim, which has a flat structure, fewer terms, and broader definitions. The list of GO Slim terms for Saccharomyces cerevisiae can be downloaded from the Gene Ontology webpage (www.geneontology.org/GO_slims/goslim_yeast.obo), whereas the gene to term mapping can be obtained from the Saccharomyces Genome Database (SGD, http://downloads .yeastgenome.org/curation/literature/go_slim_mapping.tab).

Although simpler than GO, GO Slim may still provide a functional categorization that is too detailed for many visualization purposes (e.g., 167 terms in the yeast version). To address this issue, more general annotation standards have been developed. For example, the most recent yeast genetic interaction study grouped 4414 genes into 17 functional categories (Supplementary Data File S6 in Costanzo et al. (2010)).

Similarly, it is often useful to visualize functional annotations that are more specific than GO Slim, such as, for example, individual biological pathways or protein complexes. In addition to a few systematic analyses of co-complex associations (Gavin et al. 2006; Krogan et al. 2006), several groups have curated and integrated the results of multiple experimental data sets to 
produce a compendium of protein complexes for yeast (http://downloads.yeastgenome.org/curation/ literature/go_protein_complex_slim.tab) (Pu et al. 2009; Baryshnikova et al. 2010).

\section{ACKNOWLEDGMENTS}

I thank Michael Costanzo for providing critical feedback on the manuscript.

\section{REFERENCES}

Ashburner M, Ball CA, Blake JA, Botstein D, Butler H, Cherry JM, Davis AP, Dolinski K, Dwight SS, Eppig JT, et al. 2000. Gene ontology: Tool for the unification of biology. The Gene Ontology Consortium. Nat Genet 25: $25-29$.

Atkinson HJ, Morris JH, Ferrin TE, Babbitt PC. 2009. Using sequence similarity networks for visualization of relationships across diverse protein superfamilies. PLoS One 4: e4345.

Baryshnikova A, Costanzo M, Kim Y, Ding H, Koh J, Toufighi K, Youn JY, Ou J, San Luis BJ, Bandyopadhyay S, et al. 2010. Quantitative analysis of fitness and genetic interactions in yeast on a genome scale. Nat Methods 7: 1017-1024.

Breuer K, Foroushani AK, Laird MR, Chen C, Sribnaia A, Lo R, Winsor GL, Hancock RE, Brinkman FS, Lynn DJ. 2013. InnateDB: Systems biology of innate immunity and beyond - Recent updates and continuing curation. Nucleic Acids Res 41: D1228-D1233.

Costanzo M, Baryshnikova A, Bellay J, Kim Y, Spear ED, Sevier CS, Ding H, Koh JL, Toufighi K, Mostafavi S, et al. 2010. The genetic landscape of a cell. Science 327: 425-431.

Freeman TC, Goldovsky L, Brosch M, van Dongen S, Maziere P, Grocock RJ, Freilich S, Thornton J, Enright AJ. 2007. Construction, visualisation, and clustering of transcription networks from microarray expression data. PLoS Comput Biol 3: 2032-2042.
Fruchterman TMJ, Reingold EM. 1991. Graph drawing by force-directed placement. Software 21: 1129-1164.

Gavin AC, Aloy P, Grandi P, Krause R, Boesche M, Marzioch M, Rau C, Jensen LJ, Bastuck S, Dumpelfeld B, et al. 2006. Proteome survey reveals modularity of the yeast cell machinery. Nature 440: 631-636.

Kamada T, Kawai S. 1989. An algorithm for drawing general unidirected graphs. Processing Lett 31: 7-15.

Krogan NJ, Cagney G, Yu H, Zhong G, Guo X, Ignatchenko A, Li J, Pu S, Datta N, Tikuisis AP, et al. 2006. Global landscape of protein complexes in the yeast Saccharomyces cerevisiae. Nature 440: 637-643.

Maere S, Heymans K, Kuiper M. 2005. BiNGO: A Cytoscape plugin to assess overrepresentation of gene ontology categories in biological networks. Bioinformatics 21: 3448-3449.

Myers CL, Barrett DR, Hibbs MA, Huttenhower C, Troyanskaya OG. 2006. Finding function: Evaluation methods for functional genomic data. BMC Genomics 7: 187.

Pu S, Wong J, Turner B, Cho E, Wodak SJ. 2009. Up-to-date catalogues of yeast protein complexes. Nucleic Acids Res 37: 825-831.

Smoot ME, Ono K, Ruscheinski J, Wang PL, Ideker T. 2011. Cytoscape 2.8: New features for data integration and network visualization. Bioinformatics 27: 431-432. 


\section{Exploratory Analysis of Biological Networks through Visualization, Clustering, and Functional Annotation in Cytoscape}

Anastasia Baryshnikova

Cold Spring Harb Protoc; doi: 10.1101/pdb.prot077644 originally published online March 17, 2016

\begin{tabular}{|c|c|}
\hline $\begin{array}{l}\text { Email Alerting } \\
\text { Service }\end{array}$ & Receive free email alerts when new articles cite this article - click here. \\
\hline $\begin{array}{l}\text { Subject } \\
\text { Categories }\end{array}$ & $\begin{array}{l}\text { Browse articles on similar topics from Cold Spring Harbor Protocols. } \\
\text { Bioinformatics/Genomics, general (192 articles) } \\
\text { Computational Biology (100 articles) } \\
\text { Genome-wide and Computer-based Analysis (5 articles) } \\
\text { Protein: Protein Interactions ( } 86 \text { articles) } \\
\text { Protein: Protein Interactions, general (112 articles) } \\
\text { Yeast Genetics (135 articles) }\end{array}$ \\
\hline
\end{tabular}

